\title{
Publisher Correction: Mitochondrial pyruvate carriers are required for myocardial stress adaptation
}

Yuan Zhang (D), Paul V. Taufalele (D), Jesse D. Cochran (D), Isabelle Robillard-Frayne, Jonas Maximilian Marx (D), Jamie Soto, Adam J. Rauckhorst (D), Fariba Tayyari D, Alvin D. Pewa, Lawrence R. Gray, Lynn M. Teesch, Patrycja Puchalska, Trevor R. Funari (D), Rose McGlauflin, Kathy Zimmerman, William J. Kutschke (iD, Thomas Cassier, Shannon Hitchcock, Kevin Lin, Kevin M. Kato (D), Jennifer L. Stueve (D), Lauren Haff, Robert M. Weiss (D), James E. Cox (D), Jared Rutter (D), Eric B. Taylor, Peter A. Crawford, E. Douglas Lewandowski (D), Christine Des Rosiers and E. Dale Abel (D)

Correction to: Nature Metabolism https://doi.org/10.1038/s42255-020-00288-1, published online 26 October 2020.

In the version of this article initially published, in the $y$ axes in Fig. $4 \mathrm{a}, \mathrm{b}$ ' $\mathrm{M}+1$ ' should have read ' $\mathrm{M}+i$ '. The errors have been corrected in the HTML and PDF versions of the article.

Published online: 18 November 2020

https://doi.org/10.1038/s42255-020-00322-2

(c) The Author(s), under exclusive licence to Springer Nature Limited 2020 